In Cres. Vol. $3 N^{\circ}$ 2: pp. 247-255, 2012

\title{
CONOCIMIENTO PROFESIONAL Y ACTUACIÓN DOCENTE DEL PROFESOR INNOVADOR EN EL CONTEXTO SOCIOCULTURAL*
}

\author{
PROFESSIONAL KNOWLEDGE AND INNOVATIVE TEACHER TEACHER \\ PERFORMANCE IN THE CONTEXT SOCIOCULTURAL
}

\section{Carmen Veruska Cerna Vega ${ }^{I}$}

\section{RESUMEN}

La presente investigación se realiza en torno al saber docente, manifestado a través de su narrativa. La investigación es llevada a cabo como tesis de maestría denominada "Conocimiento profesional y actuación docente del profesor innovador en el contexto socio cultural".

La problemática que motiva la realización del presente estudio es determinar el potencial innovador que presentan los profesores al momento de insertarse en el espacio de la actuación docente, para comprender la utilidad de las teorías formales en la comprensión de las situaciones que acontecen en el aula.

De este modo se determinó el conocimiento y la actuación docente de la profesional en estudio, en el Nivel Inicial, que labora en la I.E. $N^{\circ} 1617$ del Asentamiento Humano Florida Alta, del distrito de Chimbote, provincia del Santa, departamento de Ancash, teniendo en cuenta las fuentes de su formación profesional y, en especial, la formación académica, desde la perspectiva de sus propias capacidades y versiones dadas por otros actores.

Para el conocimiento profesional, el análisis se realizó sobre el material obtenido mediante la entrevista en profundidad; para la actuación docente se realizaron observaciones de clases en el aula, empleando material fílmico, reflexionando sobre las mismas, para emitir un juicio de valor y cuantificarlo.

El diseño, a partir del cual se desarrolla el trabajo de investigación, se basa en el enfoque cualitativo-cuantitativo de tipo biográfico narrativo. La lógica que se persigue es de compren-

* Recibido: 15 de mayo del 2012; aprobado: 25 de octubre del 2012.

1 Magister en Educación Mencion: En Docencia, Curriculo, e Investigación. Presidenta de la Comisión de Investigación de Educación en la Universidad Católica Los Ángeles de Chimbote. 
sión e interpretación a partir de situar el objeto de estudio: el desarrollo del conocimiento profesional del profesor, considerando que éste permite estudiar y comprender los procesos de metacognición y pensamiento de la profesora desde la perspectiva de su conocimiento y actuación profesional, y la intencionalidad que da sentido a sus pensamientos, acciones y sentimientos.

PALABRAS ClAVE: Conocimiento profesional, Actuación docente, Profesor innovador.

\begin{abstract}
This research was done about teacher knowledge, expressed through its narrative. Research is carried out as master's thesis entitled "Professional knowledge and innovative teacher's teaching performance in the socio-cultural context".

The problem that motivates the achievement of this study is to determine the potential for innovation, presented by teachers, when inserted in the space of teaching performance, to understand the usefulness of formal theories in the understanding of the situations that occur in the classroom.
\end{abstract}

Thus we determined the knowledge and teaching performance of professional study, Level, working in the IE No. 1617 of the Florida High slum district of Chimbote, Santa province, Ancash department taking into account the sources of their training and, in particular, the academic training from the perspective of their own abilities and accounts given by other actors.

For the professional knowledge, the analysis was performed on material obtained through indepth interview for the teaching performance was conducted classroom observations in the classroom, using film footage, reflecting on them, to make a value judgment and quantify.

The design, from which develops the research work is based on the qualitative-quantitative approach biographical narrative type. The logic is pursued understanding and interpretation from placing the object of study: the development of professional knowledge of teachers. Considering that it allows to study and understand the processes of metacognition and thinking from the perspective of teacher knowledge and performance, and intentionality that gives meaning to their thoughts, actions and feelings.

KEY WORDS: Professional knowledge, Teaching performance, Innovative teacher.

\title{
INTRODUCCIÓN
}

El análisis de la presente investigación sobre el conocimiento profesional y la actuación docente es producto de la investigación llevada a cabo como tesis de maestría "Conocimiento profesional y actuación docente del profesor innovador", estudio realizado en el marco del contexto sociocultural del aula, enmarcada en los estudios: conocimiento profesional del profesor, la narrativa y su actuación docente. 
La problemática que motivó el presente estudio se refiere a las potencialidades y saberes que presentan los profesores al momento de insertarse y desarrollarse en el espacio de la práctica docente. La línea de base de la problemática se refiere a la construcción del conocimiento profesional del docente, los condicionantes que hacen a su constitución y desarrollo y el papel que tiene la formación inicial en el mismo. ${ }^{1}$

El presente trabajo de investigación tuvo como propósito describir y fundamentar la naturaleza y calidad del conocimiento profesional y de la actuación docente innovadora que poseen los profesores en un determinado contexto sociocultural del aula. Así mismo, describir y argumentar, desde el análisis biográfico, la naturaleza y calidad del conocimiento profesional que poseen los profesores participantes del estudio, realizando un proceso de discriminación de experiencias educativas e identificando el grado de significatividad de las mismas como una forma de entender el conocimiento que poseen los profesores.

\section{PROBLEMA}

¿Cuál es el nivel de conocimiento profesional que poseen los profesores innovadores?, ¿Cuál es la calidad de la narrativa del profesor, con relación al conocimiento profesional que posee?, ¿Qué elementos innovadores configuran la actuación docente del profesor en el aula?

\section{OBJETIVO GENERAL}

Identificar el nivel de conocimiento profesional y actuación docente del profesor innovador en el contexto sociocultural de la I.E. N ${ }^{0} 1617$, Asentamiento Humano Florida Alta, del distrito de Chimbote, provincia del Santa, departamento de Ancash.

\section{OBJETIVOS ESPECÍFICOS}

1. Caracterizar y valorar la dimensión sustantiva del conocimiento profesional que posee la profesora innovadora.

2. Caracterizar y valorar la dimensión sintáctica del conocimiento profesional que posee la profesora innovadora.

3. Identificar y definir la calidad de la narrativa en su dimensión sustantiva en relación al conocimiento profesional de la profesora innovadora.

4. Identificar y definir la calidad de la narrativa en su dimensión sintáctica en relación al conocimiento profesional de la profesora innovadora. 


\section{MATERIAL Y MÉTODOS}

Esta investigación es de tipo cualitativo-cuantitativo, biográfica narrativa (mixto), porque hace uso del enfoque biográfico-narrativo, pues su objetivo es recopilar y sistematizar información proveniente de sus propios protagonistas, a fin de presentar planteamientos integradores cuantificarlos e interpretativos. ${ }^{2}$ Es de tipo exploratorio-descriptivo. Exploratorio, porque se realiza por contacto directo a través de la entrevista y la observación en un ámbito muestral reducido. Descriptivo, porque incluye la descripción de una situación mediante el estudio de la misma en circunstancias temporo-espacial determinada para obtener el conocimiento actualizado de la misma.

El diseño es descriptivo simple, que busca recoger e interpretar información con respecto a una situación previamente determinada que excluye el control de un tratamiento. En este tipo de diseño la muestra exige un número pequeño de sujetos; en este caso, un solo sujeto, para estudiar en detalle un aspecto de interés. La técnica de muestreo fue deliberada y las técnicas que se usaron fue un caso típico y conveniente que se considera una estrategia simple y secuencial, pues los métodos de ambos enfoques CUAN y CUAL aparecen una después del otro en un orden determinado. ${ }^{3}$

La muestra está compuesta por una profesional de la educación del Nivel Inicial, Lic. Lucy Varas Boza, de la Institución Educativa No 1617, del Asentamiento Humano Florida Alta, distrito de Chimbote, provincia del Santa, departamento de Ancash; seleccionada a partir de los criterios referidos al perfil de calidad profesional con orientación a la innovación como práctica sus realizaciones. ${ }^{4}$

\section{RESULTADOS}

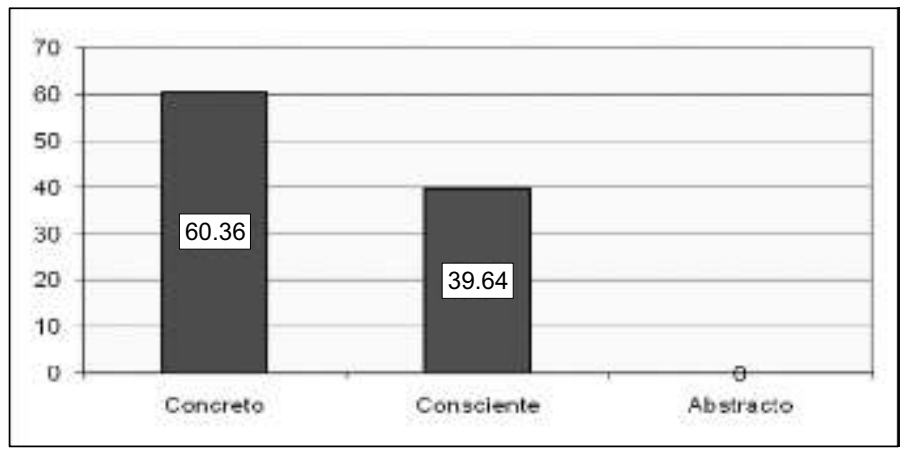

Gráfico 01. Frecuencias porcentuales del conocimiento profesional como expresión sumatoria de las dimensiones. Fuente: Tabla 01. 
Conocimiento profesional y actuación docente del profesor innovador en el contexto sociocultural

Tabla 01

FRECUENCIAS ABSOLUTAS Y PORCENTUALES DEL CONOCIMIENTO PROFESIONAL COMO EXPRESIÓN SUMATORIA DE LAS DIMENSIONES

\begin{tabular}{lcccccccc}
\hline \multirow{3}{*}{ Dimensión } & \multicolumn{10}{c}{ Niveles } \\
\cline { 2 - 11 } & \multicolumn{2}{c}{ Concreto } & \multicolumn{1}{c}{ Consciente } & \multicolumn{2}{c}{ Abstracto } & \multicolumn{2}{c}{ Total } \\
\cline { 2 - 10 } & f & $\%$ & f & $\%$ & f & $\%$ & f & \% \\
\hline Sustantiva & 34 & 60,71 & 22 & 39,29 & 0 & 0,00 & 34 & 60,71 \\
Sintáctica & 33 & 60,00 & 22 & 40,00 & 0 & 0,00 & 33 & 60,00 \\
Total conocimiento profesional & 67 & 60,36 & 44 & 39,64 & 0 & 0,00 & 67 & 60,36 \\
\hline
\end{tabular}

Fuente: Entrevista en profundidad.

Según los resultados obtenidos con relación a las dos dimensiones, se observa que la Dimensión Sustantiva del conocimiento profesional obtiene la puntuación más alta en el nivel concreto con $60,71 \%$, seguida por la Dimensión Sintáctica con $60 \%$ en el nivel concreto.

Según los datos obtenidos, el conocimiento profesional de la profesora se ubica en el nivel concreto con $60,36 \%$. La diferencia porcentual con el nivel consciente es considerable, el mismo que alcanzó 39,64\% de puntuación general; cabe precisar que en el nivel abstracto no obtiene puntuación alguna.

Tabla 02

FRECUENCIAS ABSOLUTAS Y PORCENTUALES DEL CONOCIMIENTO PROFESIONAL CALIDAD DE LA NARRATIVA COMO EXPRESIÓN SUMATORIA DE LAS DIMENSIONES

\begin{tabular}{|c|c|c|c|c|c|c|c|c|c|c|}
\hline \multirow{3}{*}{ Dimensión } & \multicolumn{8}{|c|}{ Niveles } & \multirow[b]{3}{*}{ f } & \multirow[b]{3}{*}{$\%$} \\
\hline & \multicolumn{2}{|c|}{ Descriptivo } & \multicolumn{2}{|c|}{ Analítico } & \multicolumn{2}{|c|}{ Explicativo } & \multicolumn{2}{|c|}{ Valorativo } & & \\
\hline & $\mathbf{f}$ & $\%$ & f & $\%$ & $\mathbf{f}$ & $\%$ & $\mathbf{f}$ & $\%$ & & \\
\hline Sustantiva & 274 & 40,8 & 126 & 32,1 & 28 & 7,1 & 162 & 36,2 & 590 & 31,0 \\
\hline Sintáctica & 292 & 44,2 & 131 & 34,0 & 34 & 8,8 & 165 & 37,5 & 622 & 33,3 \\
\hline Total Calidad de la Narrativa & 566 & 42,5 & 275 & 33,1 & 62 & 8,0 & 327 & 36,8 & 1212 & 32,1 \\
\hline
\end{tabular}

Fuente: Entrevista en profundidad.

La tabla 02, correspondiente a la subvariable calidad de la narrativa, expresa su mayor logro en el nivel descriptivo de la narrativa, donde alcanza una frecuencia de 566 puntos que representa un porcentaje de $42,5 \%$, que supera ampliamente a los puntajes obtenidos en los otros niveles; sin embargo, es de interés señalar el logro obtenido en el nivel valorativo, en el cual alcanza una frecuencia de 327 puntos, que representa el $36,8 \%$ del puntaje total esperado; 
así mismo, el nivel analítico alcanzó una frecuencia de 257 puntos, que representa el $33.1 \%$ del puntaje total esperado y, finalmente, el nivel explicativo alcanza el menor puntaje, con una frecuencia de 62 puntos, que representa el $8,0 \%$ del puntaje total esperado.

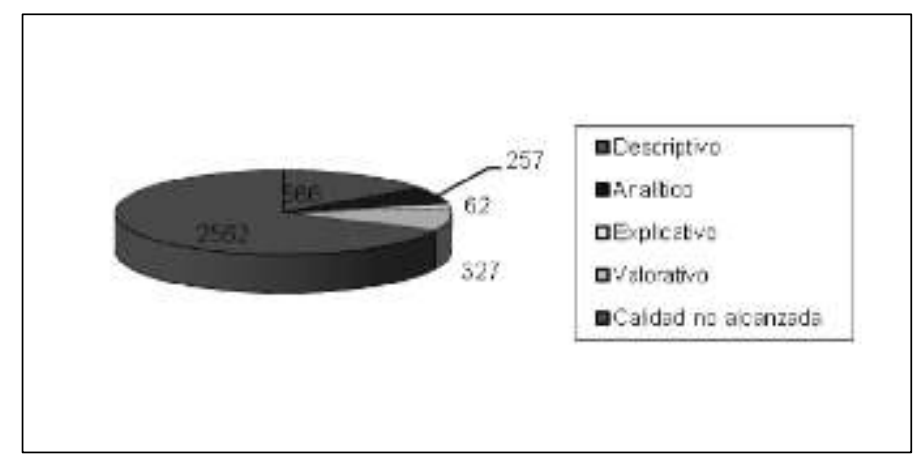

Gráfico 02. Frecuencias absolutas del conocimiento profesional calidad de la narrativa como expresión sumatoria de las dimensiones. Fuente: Tabla 01.

En el gráfico $\mathrm{N}^{\circ} 02$ se observa que, en relación a la calidad de la narrativa, la profesora alcanzó una frecuencia de 1212 puntos, lo que equivale al 32,1\% .

\section{DISCUSIÓN}

\section{A. CONOCIMIENTO PROFESIONAL}

La interpretación de los resultados obtenidos respecto a la variable conocimiento profesional, presentados en la tabla 01, refieren que la profesora Lucy Joanet Varas Boza se ubica en un nivel concreto. Tal como se ha mencionado, el nivel es de naturaleza diversificable, caracterizado fundamentalmente por la capacidad del pensamiento humano expresado en las habilidades para integrararticular el conocimiento en sus diversas facetas; así mismo, esta categoría permite al docente adecuar y reinventar estrategias didácticas.

Desde el nivel concreto y la categoría diversificable, el nivel de conocimiento alcanzado por la profesora correspondería a su característica de innovadora, por considerar que trabajó con la plena seguridad de saber lo que hacía, caracterizándose por su pensamiento descriptivo valorativo con tendencia a ser crítico frente a la acción aquel que permanentemente lo obliga a cuestionarse. ${ }^{5}$ 


\section{B. CALIDAD DE LA NARRATIVA DEL PROFESOR INNOVADOR}

Del análisis realizado a los relatos a través de las treinta y cuatro (34) unidades se obtuvieron ideas "síntesis", que configuraron los datos a los cuales se le adjudicó un valor frecuencial del procedimiento utilizado sustentando en la conversación empática entre el investigador y el profesor. Se recogieron los razonamientos teóricos y prácticos que sostienen aspectos importantes de la práctica docente. ${ }^{6}$

Este acto dialógico favoreció significativamente la construcción de un razonamiento cada vez mejor acerca de su relación e interacción con los alumnos, su rol de mediador en el acto de enseñar, aprender y su función conciliadora entre los agentes de la comunidad educativa (la escuela, la familia y la comunidad).

$\mathrm{Al}$ contrastar las hipótesis con los resultados obtenidos, se puede manifestar claramente que la profesora en estudio se ubica en el nivel descriptivo de la narrativa. La tabla 02 expone los datos totales para expresar que de un puntaje de 3774 equivalente al 100\%, calidad de la narrativa, lo que alcanzó es 1212 , haciendo un $32.1 \%$ del total esperado. De esta manera se puede definir, de acuerdo al baremo establecido, que la narrativa de la profesora está en la categoría media, en el nivel descriptivo.

Desde este punto de vista, la narrativa de la profesora que tiene una categoría media, corresponde al perfil del profesor innovador, dado que sus relatos expresan el manejo práctico- concreto de concepciones educativas, didácticas, aplicadas en el aula; es decir, relaciona sus vivencias, la teoría y la práctica en su quehacer educativo. ${ }^{7}$

\section{ACTUACIÓN DOCENTE}

La variable actuación docente, se determina tomando como punto el contexto laboral de la profesional en estudio (el aula, la institución educativa y la comunidad). Es concretamente el marco institucional donde existen una variedad de condiciones precisamente para la actuación, para el despliegue de su capacidad cognitiva y capacidad didáctica de la docente. Los elementos técnicos configurativos de esta variable son la pedagogía, el currículo y la didáctica, los cuales son analizados y contrastados a la luz de los resultados obtenidos, que nos permitirán precisar los indicadores de innovación en cada uno de los aspectos.

Para la determinación de la actuación docente, la observación como técnica para recopilar datos ha permitido configurar y evidenciar el hecho real y complejo que significa el acto mismo de enseñar y aprender. El objetivo de esta 
metodología de investigación se orienta hacia el análisis de datos cualitativos de tendencias, tipologías, regularidades o patrones y, en este caso, fundamentalmente, a la obtención de datos únicos de carácter ideográficos que desde el propósito del investigador sería la innovación. ${ }^{8}$

\section{CONCLUSIONES}

1. El conocimiento profesional de la profesora se ubica en el nivel concreto con $60,36 \%$, seguido del nivel consciente, que alcanzó 39,64\% de la puntuación general y en el nivel abstracto no obtiene puntuación alguna, lo cual permite comprobar que el nivel de conocimiento de la docente en estudio no alcanza la innovación.

2. Los resultados referidos a la dimensión sustantiva del conocimiento profesional de la profesora en estudio demuestran que el mayor puntaje está ubicado en el nivel concreto, con una frecuencia de 34 puntos, que representa el 60,71\%; y en el nivel consciente obtuvo una frecuencia de 22 puntos, que representa el 39,29\% y en el nivel abstracto obtiene una frecuencia de 0 puntos, que representa el $0,00 \%$.

3. Los resultados referidos a la dimensión sintáctica del conocimiento profesional de la profesora en estudio nos muestra que el mayor puntaje es en el nivel concreto, con una frecuencia de 33 puntos, que representa el $60 \%$. En el nivel aproximado obtuvo una frecuencia de 22 puntos, que representa el $40 \%$ y en el nivel abstracto obtiene una frecuencia de 0 puntos, que representa el $0,00 \%$.

4. En relación a la calidad de la narrativa de la profesora, se alcanzó el 32,1\%, con una frecuencia de 1212 del total esperado, equivalente a 3774 puntos. La diferencia es de $67,9 \%$, con una frecuencia absoluta de 2562 puntos, considerado como una expresión de no logro, lo cual implica que está en categoría media en la gestión pedagógica en la Educación Básica Regular, Nivel Inicial.

5. La calidad de la narrativa en el nivel descriptivo de la profesora innovadora, en relación al conocimiento profesional alcanzó una frecuencia de 566 puntos, que representa un porcentaje de $42,5 \%$; en el nivel valorativo alcanza una frecuencia de 327 puntos, que representa el $36,8 \%$ del puntaje total esperado; el nivel analítico alcanzó una frecuencia de 275 puntos, que representa el 33,1\% del puntaje total esperado; y, finalmente, el nivel explicativo alcanzó el menor puntaje, con una frecuencia de 62 puntos, que representa el $8,0 \%$ del puntaje total esperado. 
6. La calidad de la narrativa en la dimensión sustantiva se expresa su mayor logro en el nivel descriptivo, alcanzando una frecuencia de 274 puntos, equivalente a $40,8 \%$ del total esperado y el nivel explicativo obtienen la menor frecuencia con 28 puntos, alcanzando el $7,1 \%$.

7. La calidad de la narrativa en la dimensión sintáctica se expresa su mayor logro en el nivel descriptivo, alcanzando una frecuencia de 292 puntos, equivalente a $44,2 \%$ y el nivel explicativo obtiene la menor frecuencia, con 34 puntos, equivalente a $8,8 \%$.

\section{REFERENCIAS BIBLIOGRÁFICAS}

1 CASTILlo S. Formación de profesores en la educación superior desarrollo curricular y evaluación. Volumen II. (UNED) Madrid; 2006.

2 PÉREZ G. Investigación Cualitativa Investigación e Interrogantes: II Técnica y Análisis de Datos. Madrid: Editorial la Muralla S.A; 1994.

3 TOJAR J. Investigación cualitativa comprender y actuar. Madrid: Editorial La Muralla; 2006.

4 Gonzales R. Módulo de Investigación Científica. "Universidad Nacional Pedro Ruiz Gallo" Lambayeque; 2005.

5 Montero L. La Construcción del Conocimiento Profesional Docente. Argentina: Editorial Homo Sapiens; 2001.

6 Olaz Á. La Entrevista. [monografía en Internet]. 2002. [citada el 15 de setiembre 2009]. Disponible en: http://www.septem.es/tifles/libro\%200LAZres.pdf.

7 La Narrativa como Herramienta para la Reflexión Docente [serie en Internet] 2000. [citada el 11 de agosto 2009] Disponible en: http://www.sectormatematica.cl/seccion/ensayo\%20narrativa\% 20.doc.

8 TARDIF M. Los saberes del docente y su desarrollo profesional. Madrid: Narcea S.A. Ediciones; 2004. 